\title{
Multi-frequency VLBA observations of compact sources from the Peacock and Wall catalogue
}

\author{
A. Rossetti ${ }^{1}$, F. Mantovani ${ }^{1}$, D. Dallacasa ${ }^{1,2}$, C. Fanti ${ }^{1,3}$, and R. Fanti ${ }^{1,3}$ \\ ${ }^{1}$ Istituto di Radioastronomia del CNR, via Gobetti 101, 40129 Bologna, Italy \\ e-mail: rossetti@ira.cnr.it \\ 2 Dipartimento di Astronomia, Università degli Studi, via Ranzani 1, 40127 Bologna, Italy \\ 3 Dipartimento di Fisica, Università degli Studi, via Irnerio 46, 40126 Bologna, Italy
}

Received 14 July 2004 / Accepted 25 October 2004

\begin{abstract}
VLBA observations are presented for 6 compact radio sources selected from the Peacock \& Wall catalogue. From the new morphological and spectral information, 2 objects that in the Peacock and Wall catalogue are flat spectrum $(\alpha \leq 0.5)$ sources, appear to be double sided objects with linear sizes of the order of one kpc. Three are core-jet sources and the last one is still an "enigmatic" object. These data complete the sample of small double compact sources in the Peacock \& Wall catalogue and the complete list is given.
\end{abstract}

Key words. galaxies: active - radio continuum: galaxies - galaxies: quasars: general

\section{Introduction}

The Peacock \& Wall catalogue (Peacock \& Wall 1981, here after referred to as PW) lists radio sources in the Northern sky $\left(\delta>10^{\circ},|b|>10^{\circ}\right)$ stronger than $1.5 \mathrm{Jy}$ at $2.7 \mathrm{GHz}$. This is equivalent, for a spectral index $\alpha=0.7\left(S(v) \propto v^{-\alpha}\right)$, to the flux density limit of the 3CRR catalogue at $0.178 \mathrm{GHz}$ (Laing et al. 1983). Due to the high frequency selection, the PW catalogue contains a large fraction of sources with spectral index $\alpha_{0.178}^{2.7} \leq$ 0.5 , many of which are missed in the 3CRR catalogue. A large number of them are genuine flat spectrum sources showing flux density variability and core-jet radio structure on scales from tens of $\mathrm{pc}$ to $\mathrm{kpc}^{1}$ and beyond. In a number of cases, instead, the spectrum is flat at $\approx 2 \mathrm{GHz}$, inverted at lower frequencies and steepens at higher frequencies, while the radio structure is on sub-kpc scales. These sources, called GHz Peaked-Spectrum (GPS) sources, are under-represented in low frequency selected samples and their difference from the "truly flat spectrum compact sources" was emphasized by the extensors of the catalogue themselves (Peacock \& Wall 1982).

GPSs form a continuous class of sources with the Compact Steep-Spectrum (CSS) sources, sizes up to $20 \mathrm{kpc}$ and transparent synchrotron spectrum flattening below a few hundred MHz). Indeed, it has been shown (Fanti et al. 1990 and O'Dea $\&$ Baum 1997) that, when plotted on a "rest-frame turnover frequency - linear size" $\left(v_{\text {turn }}-L S\right)$ diagram, GPSs and CSSs form a continuous sequence and show that the turnover frequency is a fair estimator of their total linear size.

\footnotetext{
${ }^{1} H_{0}=100 h \mathrm{~km} \mathrm{~s}^{-1} \mathrm{Mpc}^{-1}, q_{0}=0.5$
}

A complete sample of GPSs with structural and polarization information has been presented by Stanghellini et al. (1998). For a review on CSS/GPS properties see O'Dea (1998).

A large fraction of CSSs and GPSs have a two-sided radio structure with respect to the radio core. They are named CSOs (Compact Symmetric Objects) and MSOs (Medium Symmetric Objects) according to their sub-kpc or over-kpc size. These sources are an important tool to investigate radio source evolution, since they are now interpreted as the young phase of the powerful large size (hundreds of $\mathrm{kpc}$ ) radio sources (see, e.g., Phillips \& Mutel 1982; Carvalho 1985; Fanti et al. 1995; Readhead et al. 1996b; Snellen et al. 2000, 2003). The expansion velocities recently measured for a dozen CSOs (Owsianik \& Conway 1998; Polatidis \& Conway 2003) and the determination of the radiative ages of a large number of MSOs (Murgia et al. 1999; Murgia 2003) have provided strong support for this scenario.

A sample of CSOs and MSOs suitable for statistical studies selected from the PW catalogue with the criterion $\alpha_{2.7}^{5.0} \gtrsim 0.5$ has been studied by Spencer et al. (1989), Fanti et al. (1990) and Dallacasa et al. (1995, 2004). The spectral selection they applied, while selecting CSOs and MSOs which peak below about $2 \mathrm{GHz}$ (hence expected to be typically larger than few hundred pc from the $v_{\text {turn }}-L S$ plot), would miss smaller size sources with turnover frequency between 2 and $5 \mathrm{GHz}$. These sources would remain hidden among the truly flat spectrum core-jet sources and can be recognized only by means of high resolution observations. Several PW sources with $\alpha \leq$ 0.5 were already imaged with the appropriate resolution and showed up as CSOs (for instance 0710+439 and 2352+495, 
Table 1. General source parameters: Col. 1: source name; Col. 2: optical identification (Id), $\mathrm{G}=$ galaxy, $\mathrm{Q}=$ quasar; Col. 3: redshift $(z)$ from Johnston et al. (1995); Col. 4: $V$ magnitude $\left(m_{v}\right)$ from Johnston et al. (1995); Col. 5: flux density at $5 \mathrm{GHz}$ from Johnston et al. (1995); Col. 6: flux density at $8.4 \mathrm{GHz}$ from Patnaik et al. (1992); Col. 7: linear Size $(L S)$ from previous VLBI observations.

\begin{tabular}{|c|c|c|c|c|c|c|}
\hline $\begin{array}{l}\text { Source } \\
\text { (1) }\end{array}$ & (2) & $\begin{array}{l}z \\
(3)\end{array}$ & $\begin{array}{l}m_{v} \\
\text { (4) }\end{array}$ & $\begin{array}{l}S_{5} \\
\mathrm{Jy} \\
(5)\end{array}$ & $\begin{array}{l}S_{8.4} \\
\text { Jy } \\
(6)\end{array}$ & $\begin{array}{l}L S \\
\text { pc } \\
(7)\end{array}$ \\
\hline $0133+476$ & $\mathrm{Q}$ & 0.859 & 19.0 & 3.3 & 1.70 & $>15$ \\
\hline $0153+744$ & Q & 2.338 & 16.0 & 1.5 & 0.91 & $\sim 12$ \\
\hline $0202+149$ & $?$ & $0.405^{(1)}$ & 21.9 & 2.4 & $2.27^{(2)}$ & $\sim 6$ \\
\hline $0859+470$ & Q & 1.462 & 18.7 & 1.8 & 0.96 & $<3$ \\
\hline $0945+664$ & $\mathrm{G}$ & - & 21.6 & $1.4^{(3)}$ & 0.78 & - \\
\hline $0954+556$ & Q & 0.909 & 17.7 & 2.3 & 1.55 & - \\
\hline
\end{tabular}

(1) Perlman et al. (1998), AJ, 115, 1253.

(2) Wright \& Otrupcek, PKS Catalogue (1990).

(3) Becker et al. (1991), ApJS, 75, 1.

Wilkinson et al. 1994; OQ208 Stanghellini et al. 1997), but, at the time we started this investigation, for six of them the available radio structure information did not allow, in our opinion, a secure radio morphological classification.

In this paper, we present the results of the VLBA observations of the 6 mentioned radio sources.

The source list and observations are presented in Sect. 2, while in Sect. 3 we describe the data reduction procedures. In Sect. 4 we discuss the morphological and spectral properties of each source. In Sect. 5 we report the complete list of CSOs/MSOs from the PW catalogue, with the relevant data. Conclusions are given in Sect. 6.

\section{The sample}

In order to find new CSSs/GPSs within the PW flat spectrum population, we searched in the literature for both sub-arcsecond structure information (Fey et al. 1996, 1997; Xu et al. 1995; Kellermann et al. 1998) and spectral shape (Kühr et al. 1981; Herbig \& Readhead 1992). For a large fraction of these flat spectrum sources they were available and allowed a firm classification as core-jet sources.

However, for 6 objects we could not find any information or the existing images did not ăallow, in our opinion, a secure classification. In some of these sources flux density measurements showed a moderate steepening of the radio spectrum above $5 \mathrm{GHz}$, while the others showed a flat spectrum over the whole explored range of frequencies.

The sources are listed in Table 1. Four of them are associated with quasars and one is a radio galaxy. The last one $(0202+149)$ is still an "enigmatic" object (see Sect. 4).

\section{Observations and data reduction}

The VLBA observations were carried out in 4 different runs, from May 1999 to November 1999 (see Table 2).
Table 2. Observation data: Col. 1: source name; Col. 2: frequency; Col. 3: date of observation; Col. 4: missing antennas (empty = 10 VLBA antennas); Col. 5: shortest projected baseline; Col. 6: beam angular size of major, minor axis and position angle of the major axis; Col. 7: rms noise level measured on the images.

\begin{tabular}{|c|c|c|c|c|c|c|c|c|}
\hline Source & $\begin{array}{r}v \\
\mathrm{GHz} \\
(2)\end{array}$ & Obs. date & $\begin{array}{c}\text { Missing } \\
\text { antennas } \\
\text { (4) }\end{array}$ & $\begin{array}{c}u_{\min } \\
\mathrm{M} \lambda \\
(5)\end{array}$ & $\begin{array}{c}\text { mas } \\
-\end{array}$ & $\begin{array}{c}\text { Beam } \\
\text { mas } \\
(6)\end{array}$ & $\begin{array}{l}\circ \\
-\end{array}$ & $\begin{array}{c}\text { Noise } \\
\text { mJy/beam } \\
(7)\end{array}$ \\
\hline \multirow[t]{3}{*}{$0133+476$} & 1.7 & $02 / 05 / 1999$ & $\overline{\mathrm{NL}}$ & 1.1 & 6.7 & 4.0 & -3 & 0.12 \\
\hline & 4.8 & 02/05/1999 & NL & 2.5 & 2.6 & 2.0 & -8 & 0.07 \\
\hline & 8.4 & 20/06/1999 & MK & 4.0 & 1.5 & 1.1 & 24 & 0.20 \\
\hline \multirow[t]{2}{*}{$0153+744$} & 4.8 & 02/05/1999 & NL & 2.5 & 1.3 & 1.2 & 78 & 0.13 \\
\hline & 15 & 20/06/1999 & MK & 8.3 & 0.7 & 0.5 & -64 & 0.08 \\
\hline \multirow[t]{2}{*}{$0202+149$} & 8.4 & 20/06/1999 & MK & 5.1 & 2.4 & 1.4 & -15 & 0.12 \\
\hline & 15 & 20/06/1999 & MK & 9.2 & 1.1 & 0.6 & -22 & 0.35 \\
\hline $0859+470$ & 15 & $13 / 11 / 1999$ & & 9.5 & 0.9 & 0.6 & -16 & 0.09 \\
\hline \multirow[t]{3}{*}{$0945+664$} & 1.7 & 03/11/1999 & & 0.8 & 7.2 & 6.3 & -7 & 0.13 \\
\hline & 4.8 & 03/11/1999 & & 2.4 & 18 & 13 & 80 & 0.38 \\
\hline & 15 & $13 / 11 / 1999$ & & 7.5 & 0.8 & 0.6 & -8 & 0.06 \\
\hline \multirow[t]{3}{*}{$0954+556$} & 1.7 & 03/11/1999 & & 1.1 & 7.3 & 4.3 & 12 & 0.13 \\
\hline & 4.8 & 03/11/1999 & & 2.5 & 2.1 & 1.8 & -18 & 0.08 \\
\hline & 15 & $13 / 11 / 1999$ & & 7.1 & 0.9 & 0.6 & -4 & 0.06 \\
\hline
\end{tabular}

According to the information available in the literature, each source was observed at one or more of the following frequencies - $1.7 \mathrm{GHz}, 4.8 \mathrm{GHz}, 8.4 \mathrm{GHz}$ and $15 \mathrm{GHz}$ - with a recording band-width of $32 \mathrm{MHz}$ at $64 \mathrm{Mbps}$, for about 2-3 h divided into scans of $44 \mathrm{~min}$. The shortest baseline is Pie Town - Los Alamos and this may have caused some flux density losses in components with angular size $\gtrsim 20$ mas at $15 \mathrm{GHz}$ or $\sim 200$ mas at $1.7 \mathrm{GHz}$.

The data were correlated with the NRAO VLBA processor at Socorro and reduced using AIPS (Astronomical Image Processing System). System temperatures measured at the antennas during the observations and gain-elevation tables provided by NRAO were used for amplitude calibrations. Amplitudes for calibrator sources (3C 84, DA193) were found to be generally consistent within $3 \%$ of expectations. Data were edited with standard procedures. Global fringe-fitting was performed using the AIPS task FRING with solution intervals of about $4 \mathrm{~min}$ at $1.7-4.8-8.4 \mathrm{GHz}$ and $1 \mathrm{~min}$ at $15 \mathrm{GHz}$. All sources provided fringes with high signal-to-noise ratio on all baselines except $0945+664$ for which the task FRING could not find solutions with signal to noise ratio $\geq 5$ at any of the three frequencies for any solution interval. For this source we successfully applied the delay and rate solutions found for $0954+556\left(\sim 11^{\circ}\right.$ apart from $\left.0945+664\right)$, observed in adjacent scans.

Images were obtained with the AIPS task IMAGR after a number of phase self-calibration iterations using a decreasing solution interval, starting with a few minutes (6 or 4 ) down to $0.5 \mathrm{~min}$, ending with a final step of amplitude self-calibration with a solution interval longer than the scan length (44 min) in order to remove residual systematic errors. This latter step was applied only if the gain corrections were $<3 \%$.

The source total flux densities were obtained by integration over the source images by means of AIPS task TVSTAT. For moderately compact components, Gaussian fits were performed with the AIPS task JMFIT, which provides positions, angular sizes and total flux density. Occasionally we made use of images at moderate super-resolution. Component positions 
Table 3. Observed source parameters derived from images in Fig. 1: Col. 1: source name; Col. 2: observing frequency (GHz); Col. 3: source total flux density (Jy) measured by using TVSTAT; Cols. 4, 5: Largest Angular Size (LAS, mas) and Largest Linear Size (LLS, pc) (for the galaxy 0945+664 we assumed $z=1$ ); Col. 6: sub-component label; components with an “*” are extended; Cols. 7, 8: distance $r$ (mas) and PA $\left({ }^{\circ}\right)$ from the reference component; Col. 9: deconvolved angular size of major and minor axis (mas) and the major axis position angle (degrees) from gaussian fit; for extended components linear sizes are derived from the images; Cols. 10, 11: peak flux density (mJy/beam) and integrated flux density (mJy); Cols. 12, 13: low and high frequency spectral indices.

\begin{tabular}{|c|c|c|c|c|c|c|c|c|c|c|c|c|c|c|}
\hline $\begin{array}{c}\text { Source } \\
\text { (1) }\end{array}$ & $\begin{array}{c}v \\
\mathrm{GHz} \\
(2)\end{array}$ & $\begin{array}{r}S_{\text {tot }} \\
\text { Jy } \\
(3)\end{array}$ & $\begin{array}{r}\text { LAS } \\
\text { mas } \\
(4)\end{array}$ & $\begin{array}{c}\mathrm{LLS} \\
\mathrm{pc} \mathrm{h}^{-1} \\
(5)\end{array}$ & $\begin{array}{c}\text { Comp. } \\
\text { (6) }\end{array}$ & $\begin{array}{c}r \\
\text { mas } \\
(7)\end{array}$ & $\begin{array}{c}\mathrm{PA} \\
\circ \\
(8)\end{array}$ & $\begin{array}{r}\text { Maj } \\
\text { mas } \\
-\end{array}$ & $\begin{array}{r}\text { Min } \\
\text { mas } \\
(9)\end{array}$ & $\begin{array}{c}\mathrm{PA} \\
\circ \\
-\end{array}$ & $\begin{array}{r}S_{\mathrm{p}} \\
\mathrm{mJy} / \mathrm{b} \\
(10)\end{array}$ & $\begin{array}{r}S_{\mathrm{t}} \\
\mathrm{mJy} \\
(11)\end{array}$ & $\begin{array}{l}\alpha_{1 \mathrm{o}} \\
(12)\end{array}$ & (13) \\
\hline \multirow[t]{7}{*}{$0133+476$} & 1.7 & 1.39 & 33 & 137 & $\mathrm{~A}$ & & & 3.37 & 1.43 & 157 & 1080.4 & 1276.9 & -0.37 & -0.55 \\
\hline & & & & & $\mathrm{Ext}^{*}$ & & & 50 & 21 & & - & 116.3 & & \\
\hline & 4.8 & 1.88 & 20 & 83 & A & & & 0.95 & 0.29 & 150 & 1678.3 & 1800.4 & & \\
\hline & & & & & Ext $^{*}$ & & & 27 & 12 & & - & 79.6 & & \\
\hline & 8.4 & 2.58 & 4 & 17 & A1 & 0 & 0 & 0.47 & 0.12 & 149 & 2286.4 & 2474.6 & & \\
\hline & & & & & A 2 & $2.5 \pm 0.15$ & -38 & 3.61 & 1.48 & 134 & 20.3 & 99.6 & & \\
\hline & & & & & A3 & $4.3 \pm 0.2$ & -38 & $<1.03$ & & & 5.9 & 6.9 & & \\
\hline \multirow{11}{*}{$0153+744$} & 4.8 & 1.04 & 10 & 39 & A & 0 & 0 & 1.50 & 0.60 & 119 & 196.4 & 348.8 & & 0.29 \\
\hline & & & & & B & $10.1 \pm 0.3$ & 156 & 1.68 & 0.65 & 118 & 270.8 & 530.2 & & 1.70 \\
\hline & & & & & $\mathrm{C}$ & $7.4 \pm 0.4$ & & 3.43 & 1.10 & 154 & 20.4 & 85.4 & & 2.04 \\
\hline & & & & & D & $4.0 \pm 0.4$ & & 1.40 & 0.95 & 159 & 8.9 & 11.6 & & \\
\hline & & & & & E & $9.3 \pm 0.4$ & & $<1.00$ & & & 46.4 & 54.0 & & 2.16 \\
\hline & 15 & 0.36 & 10 & 39 & A1 & 0 & 0 & $<0.19$ & & & 186.7 & 195.3 & & \\
\hline & & & & & A2 & $0.61 \pm 0.1$ & 69 & 1.00 & 0.27 & 98 & 36.4 & 73.5 & & \\
\hline & & & & & A3 & $1.21 \pm 0.1$ & 81 & 0.60 & 0.20 & 139 & 5.9 & 26.4 & & \\
\hline & & & & & B & & 81 & 1.72 & 0.85 & 122 & 15.3 & 77.8 & & \\
\hline & & & & & $\mathrm{C}$ & & & 2.40 & 1.17 & 168 & 1.2 & 7.8 & & \\
\hline & & & & & E & & & 1.35 & 0.99 & 36 & 1.3 & 6.0 & & \\
\hline \multirow[t]{5}{*}{$0202+149$} & 8.4 & 2.07 & $\sim 5$ & 16 & A & 0 & 0 & 0.82 & 0.23 & 124 & 1648.2 & 1831.1 & & 0.32 \\
\hline & & & & & $\mathrm{D}$ & $4.8 \pm 0.2$ & -50 & 2.81 & 2.12 & 20 & 88.8 & 254.1 & & 0.58 \\
\hline & 15 & 1.63 & $\sim 5$ & 16 & A & & & 0.75 & 0.15 & 132 & 1151.7 & 1477.1 & & \\
\hline & & & & & D1 & $4.9 \pm 0.15$ & -60 & 1.04 & 0.42 & 88 & 24.7 & 53.0 & & \\
\hline & & & & & D2 & $5.1 \pm 0.15$ & -45 & 1.83 & 0.92 & 61 & 20.4 & 82.8 & & \\
\hline \multirow[t]{4}{*}{$0859+470$} & 15 & 0.64 & $\sim 5$ & 21 & A1 & 0 & 0 & $<0.74$ & & & 388.4 & 404.3 & & \\
\hline & & & & & A 2 & $0.56 \pm 0.15$ & -15 & $<1.81$ & & & 92.1 & 107.9 & & \\
\hline & & & & & A3 & $1.98 \pm 0.2$ & -10 & 2.07 & 0.67 & 169 & 15.7 & 63.4 & & \\
\hline & & & & & B & $4.36 \pm 0.4$ & 4 & 1.78 & 1.11 & 137 & 13.3 & 67.2 & & \\
\hline \multirow[t]{3}{*}{$0945+664$} & 1.7 & 0.44 & 1500 & 6370 & $\mathrm{~A}^{*}$ & & & 119 & 49 & & 20.9 & 316.4 & & \\
\hline & & & & & $\mathrm{B}^{*}$ & & & 73 & 63 & & 4.6 & 126.1 & & \\
\hline & 4.8 & 0.26 & & 510 & $\mathrm{~A}^{*}$ & & & 94 & 78 & & 77.2 & 255.4 & & \\
\hline \multirow[t]{5}{*}{$0954+556$} & 1.7 & 1.82 & $\sim 80$ & 340 & $\mathrm{~A}^{*}$ & & & 38 & 26 & & 260.7 & 975.3 & 0.42 & 0.88 \\
\hline & & & & & $\mathrm{B}^{*}$ & & & 62 & 54 & & 69.8 & 842.5 & 0.49 & 1.62 \\
\hline & 4.8 & 0.87 & $\sim 45$ & 190 & $\mathrm{~A}^{*}$ & & & 28 & 11 & & 63.6 & 531.9 & & \\
\hline & & & & & $\mathrm{B}^{*}$ & & & 37 & 20 & & 8.0 & 338.8 & & \\
\hline & 15 & 0.21 & $\sim 45$ & 190 & $\mathrm{~A}^{*}$ & & & 15 & 4 & & 10.6 & 184.4 & & \\
\hline
\end{tabular}

are given with respect to the brightest component in the image, in order to allow a comparison with similar data at other epochs and search for proper motions. As JMFIT gives errors on the various parameters which tend to be unrealistically small, we did not use them. Instead we estimated empirically the parameter uncertainties from variations of the parameters during the model fitting process.

For many resolved sources no positions are given and the angular sizes are taken from the lowest reliable contours in the images.

Images for spectral index information (not shown) were obtained at the different frequencies using the $u v$-range between the shortest baseline at high frequency and the longest baseline at low frequency and convolving to the lowest resolution.
Alignment of the images was obtained by using a compact feature as a reference. Using the package SYNAGE++ (Murgia \& Fanti 1996), which allows one to interactively select different source regions on the images, we obtained local spectral index values between pairs of frequencies ( $\alpha_{\mathrm{lo}}$ and $\alpha_{\mathrm{hi}}$ between low and high frequency pairs respectively, in Table 3 ).

Images of total intensity are shown in Fig. 1 and source parameters are given in Table 3.

\section{Comments on individual sources}

0133+476. The source integrated spectrum (Kühr et al. 1981 ) is flat with a $20-30 \%$ variability at frequencies higher than $1 \mathrm{GHz}$. From earlier VLBI observations (Pearson \& Readhead 1981; Polatidis et al. 1995) there were indications for 

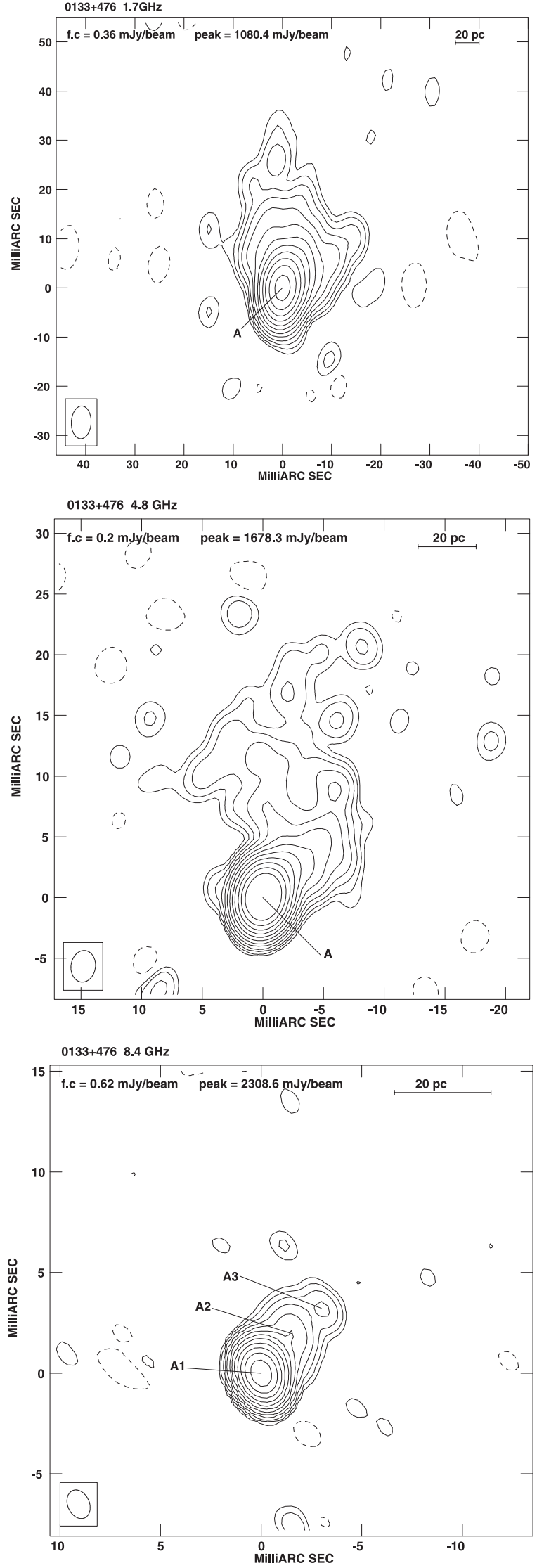

Fig. 1. VLBA images: the first contour (f.c.) is generally three times the rms noise level on the image; contour levels increase by a factor of 2; the restoring beam is shown in the bottom left corner of each image.
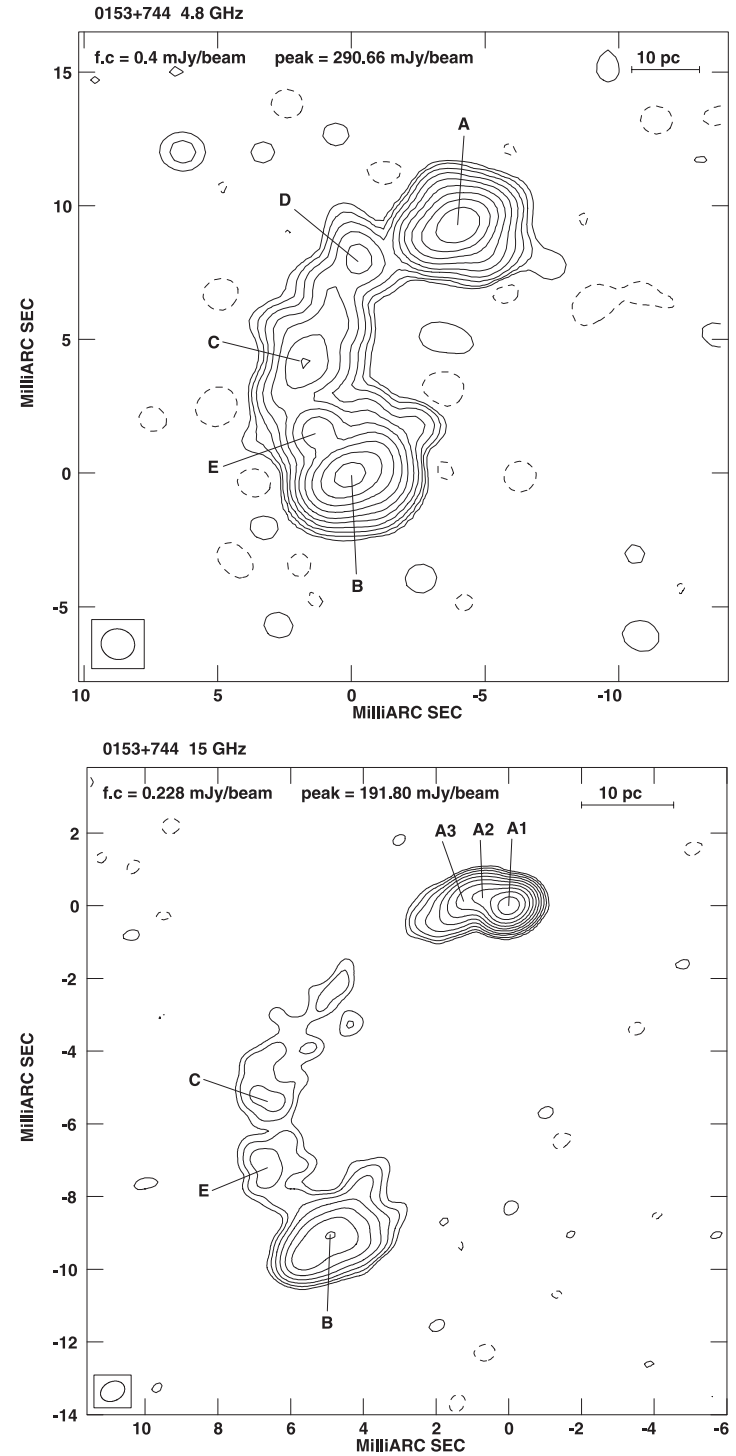

Fig. 1. continued.

classifying the source as a core-jet. At $15 \mathrm{GHz}$ (Kellermann et al. 1998) the source shows a very bright and compact component plus a fainter secondary one at a distance $\approx 3$ mas in PA $\sim-45^{\circ}$, with a very large flux density ratio and some weaker emission in between. Although the authors classified it as a single sided source, due to its similarity with OQ208 which is a CSO with very unequal flux density components, we decided to investigate it further at several frequencies.

Our images at 1.7, 4.8 and 8.4 GHz show without any doubt that the structure is indeed of the core-jet type. The radio emission is dominated by a bright compact component (component $A 1$ at $8.4 \mathrm{GHz}$ ) and a jet in $\mathrm{PA} \sim-45^{\circ}$ which diffuses, as seen often in many core-jet sources, into a sort of tail with several wiggles, seen at $5 \mathrm{GHz}$ and $1.7 \mathrm{GHz}$ North of the source.

The images, made with the same $u v$-range and restoring beam as the $1.7 \mathrm{GHz}$ observation, allowed us to obtain the spectral index of component $A$, which is only marginally resolved at $1.7 \mathrm{GHz}$. We find $\alpha_{1.7}^{4.8}=-0.37, \alpha_{4.8}^{8.4}=-0.55$, the spectral index being less inverted at low frequency probably because of 


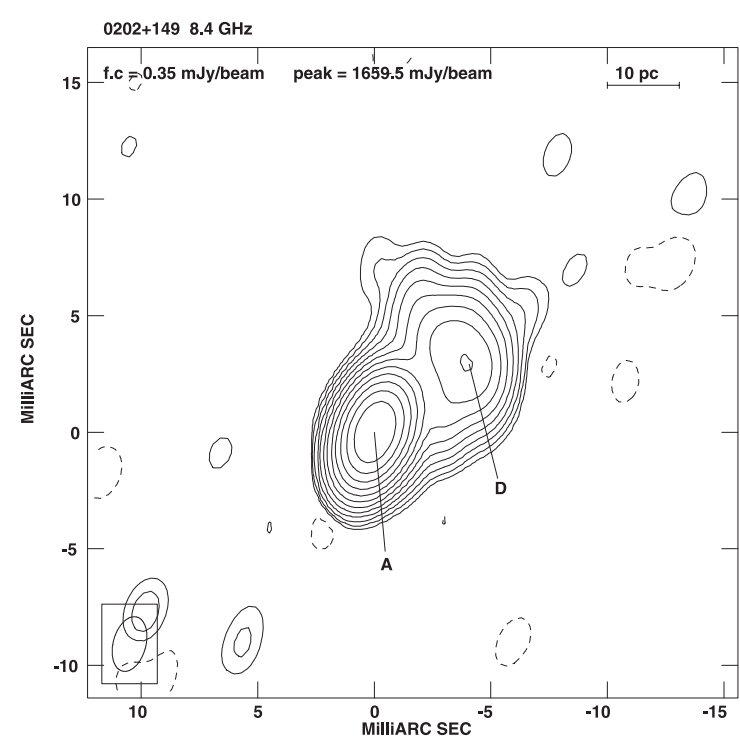

Fig. 1. continued.

a larger contribution to the total flux density from the jet/tail emission.

We compared the integrated flux densities in our images with those from the University of Michigan Radio Astronomy Observatory (UMRAO) database. Due to the source variability we took data close in time to our observing period ( \pm 2 months). At $4.8 \mathrm{GHz}$ our flux density is within $3 \%$ of what is expected, while at $8.4 \mathrm{GHz} \approx 10 \%$ of the total flux density is missing in our VLBA image, clearly due to the absence of short $u v$ spacings. The integrated spectrum of the source is definitely inverted between 1.7 and $8.4 \mathrm{GHz}$, so that the source, at our observing epoch, would be classified as a High Frequency Peaker (Dallacasa 2003).

Comparing our $8.4 \mathrm{GHz}$ flux density of component $A 1$ with the peak flux density of the bright component in the $15 \mathrm{GHz}$ map of Kellermann et al. (1998) we get an estimate of the spectral index $\alpha_{8.4}^{15} \leq 0.3$. This would suggest that the spectrum peaks somewhere in between these two frequencies. However we should consider that the two images were taken about two years apart so that the result may be affected by variability.

Component $A 2$ is likely to be the faint component in the $15 \mathrm{GHz}$ image of Kellermann et al. (2004), as the separations from the brightest component are similar in the two images. Kellermann et al. (2004) analyse the separation between $A 1$ and $A 2$ at $15 \mathrm{GHz}$, from 1994.5 to 1999.0 , and give a proper motion of $0.04 \pm 0.01 \mathrm{mas} / \mathrm{yr}\left(\beta_{\mathrm{app}}=1.01 \pm 0.25\right.$ with the cosmological parameters we use). The separation we measure $(2.5 \pm 0.2$ mas) is lower than the extrapolation of their proper motion at our epoch at a 1.8 sigma level. This difference, although not significant, may be due to spectral effects within $A 1$ (see e.g. Marcaide \& Shapiro 1984), as our image is at a frequency lower than theirs. Alternatively it may indicate that the true proper motion may be smaller than the one they reported.

$\mathbf{0 1 5 3 + 7 4 4}$. This source was studied earlier by Hummel et al. $(1988,1997)$, at several frequencies. Images at 2.4 and $8.5 \mathrm{GHz}$ can be found in Fey et al. (1997). The source integrated spectrum (Kühr et al. 1981) is flattish $(\alpha \approx 0.3)$ with

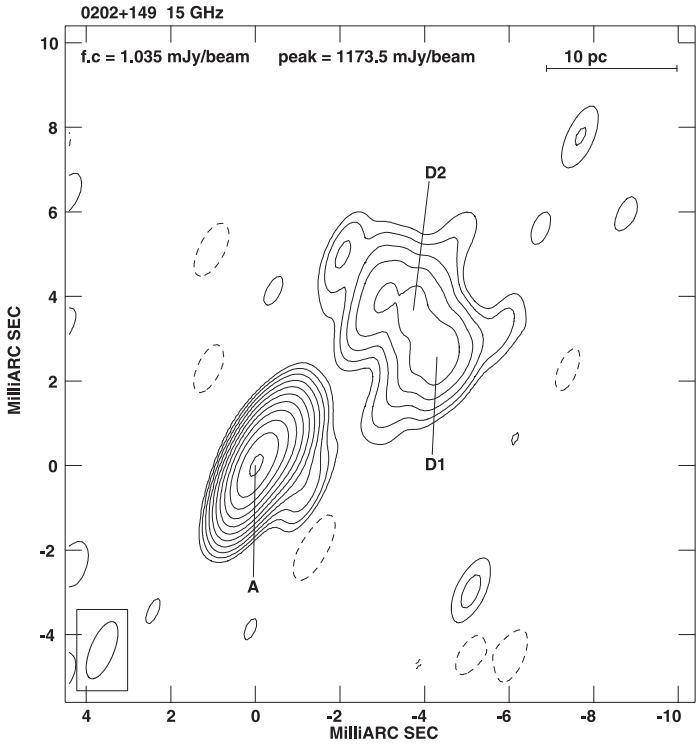

some evidence of variability. The radio emission at $\mathrm{cm}$ wavelengths is dominated by two main components, $A$ and $B$ with a knotty "bridge" of emission (components $C, D, E$ as labelled in Hummel et al. 1997). At low resolution and lower dynamic range the source resembles a small double with components of similar flux densities. In our $15 \mathrm{GHz}$ image component $A$ is resolved into three components plus a diffuse and faint outer emission region, with the brightest component at the western edge, giving the appearance of a core-jet structure, in agreement with what found by Hummel et al. (1997) at $22 \mathrm{GHz}$.

Comparing our $15 \mathrm{GHz}$ map with that of Hummel et al. (1997) at $22 \mathrm{GHz}$ we find no significant motion for component $A 2$, while component $A 3$ may have moved away from $A 1$ by $0.2 \pm 0.1$ mas over 6.6 years, leading to a proper motion of $0.035 \pm 0.017 \mathrm{mas} / \mathrm{yr}$ and an apparent velocity $\beta_{\text {app }}=1.5 \pm 0.7$.

From the comparison of our $4.8 \mathrm{GHz}$ measurements with those reported by Hummel et al. $(1988,1997)$, we find that component $A$ dropped by about a factor of two in flux density after 1990. It is likely that similar drops have occurred at other wavelengths as well so that it is not possible to determine a reliable spectral index of component $A$ over a broad frequency range from literature data taken at different epochs. From our own nearly simultaneous observations a spectral in$\operatorname{dex} \alpha_{4.8}^{15} \approx 0.29$ is derived at the resolution of the $4.8 \mathrm{GHz}$ image. We find a spectral index $\alpha_{4.8}^{15}=-0.19$ at the position of component $A 1$ which steepens away toward the East of component $A$ up to $\alpha_{4.8}^{15} \approx 1.2$.

Component $B$ shows a complex structure consisting of resolved steep-spectrum emission, embedded in a more diffuse component. From a comparison with Hummel et al. (1997) there is no evidence for flux density variability. From our flux densities we get a spectral index $\alpha_{4.8}^{15} \approx 1.7$. The data of Hummel et al. (1988) at $1.7 \mathrm{GHz}$ and of Fey et al. (1997) at $2.3 \mathrm{GHz}$, taken at lower resolutions, imply that the spectrum flattens significantly at lower frequencies $\left(\alpha_{1.7}^{4.8} \approx 0.5\right)$. Also, components $C$ and $E$ seem to have a steep high frequency spectrum. Hummel et al. (1997) have examined the separation 


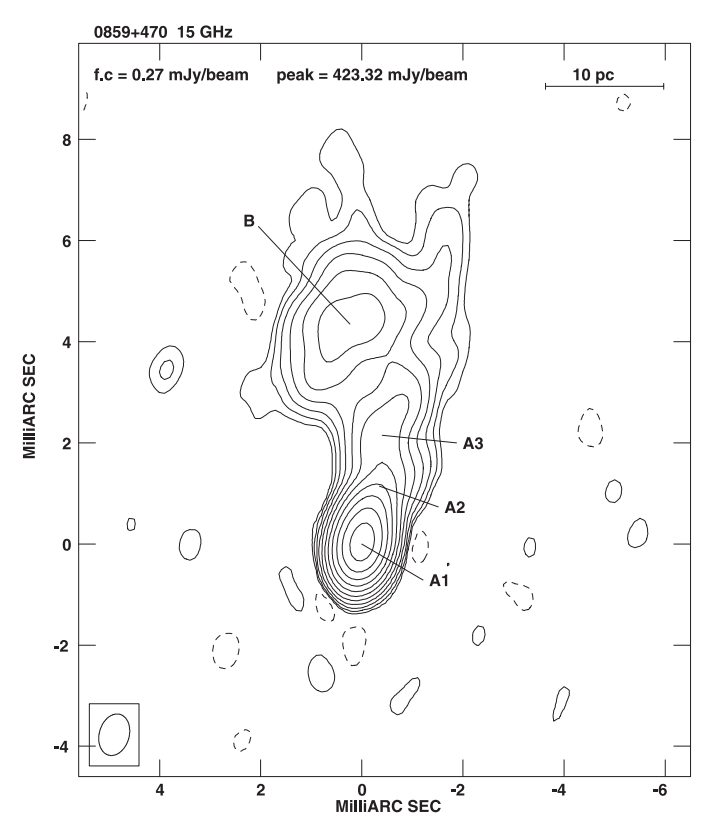

Fig. 1. continued.

of components $A$ and $B$ at several epochs and concluded that it has not changed over a 10 year interval. Our data confirm that the separation is still unchanged over 20 years giving a proper motion of $0.008 \pm 0.007 \mathrm{mas} / \mathrm{yr}$, corresponding to $\beta_{\text {app }}=0.36 \pm 0.31$.

0202+149. There is some confusion in the literature about the classification of the optical counterpart and redshift. By detecting two emission features in the optical spectrum, this object has been classified as quasar, with $z=0.833$ (Stickel et al. 1996). Comastri et al. (1997) report a redshift of 1.202. The most recent information is from Perlman et al. (1998), who published an optical spectrum which shows several narrow emission lines (e.g., $\mathrm{H} \alpha, \mathrm{H} \beta$, [OIII] Mg II), typical of low ionization narrow line radio galaxies and derived $z=0.405$. We take this as the most reliable red-shift. Stickel et al. (1996) and Impey \& Tapia (1990) report respectively red and visual magnitudes $m_{R} \approx 21.3$ and $m_{v} \approx 20.9$. Absolute magnitudes would be $M_{R} \approx-20.4$ and $M_{v} \approx=-20.8$, rather faint for a quasar. Fugmann \& Meisenheimer (1988) describe the optical spectrum as a power law with spectral index $\alpha \approx 2.3$. They also show that the infrared spectrum is consistent with the same spectral index, although the source is very variable at these wavelengths. The source is identified with a $\gamma$-ray source (von Montigny et al. 1995).

The source has a flattish radio spectrum $(\alpha \approx 0.3)$ with some variability across the whole radio band.

In the image at $15 \mathrm{GHz}$ by Kellermann et al. (1998) the source shows a very bright compact component $(A)$ plus a diffuse component $(D)$ at $\approx 5$ mas distance. In between the two, at $\mathrm{PA} \approx-80^{\circ}$ and closer to the bright component, another fainter and compact component is seen. Although the authors described the source as a core-jet we thought that it could be an asymmetric double with a weak core and therefore worth observing again. Additional images at 2.3 and $8.5 \mathrm{GHz}$ from geodetic VLBI observations (Piner \& Kingham 1998;
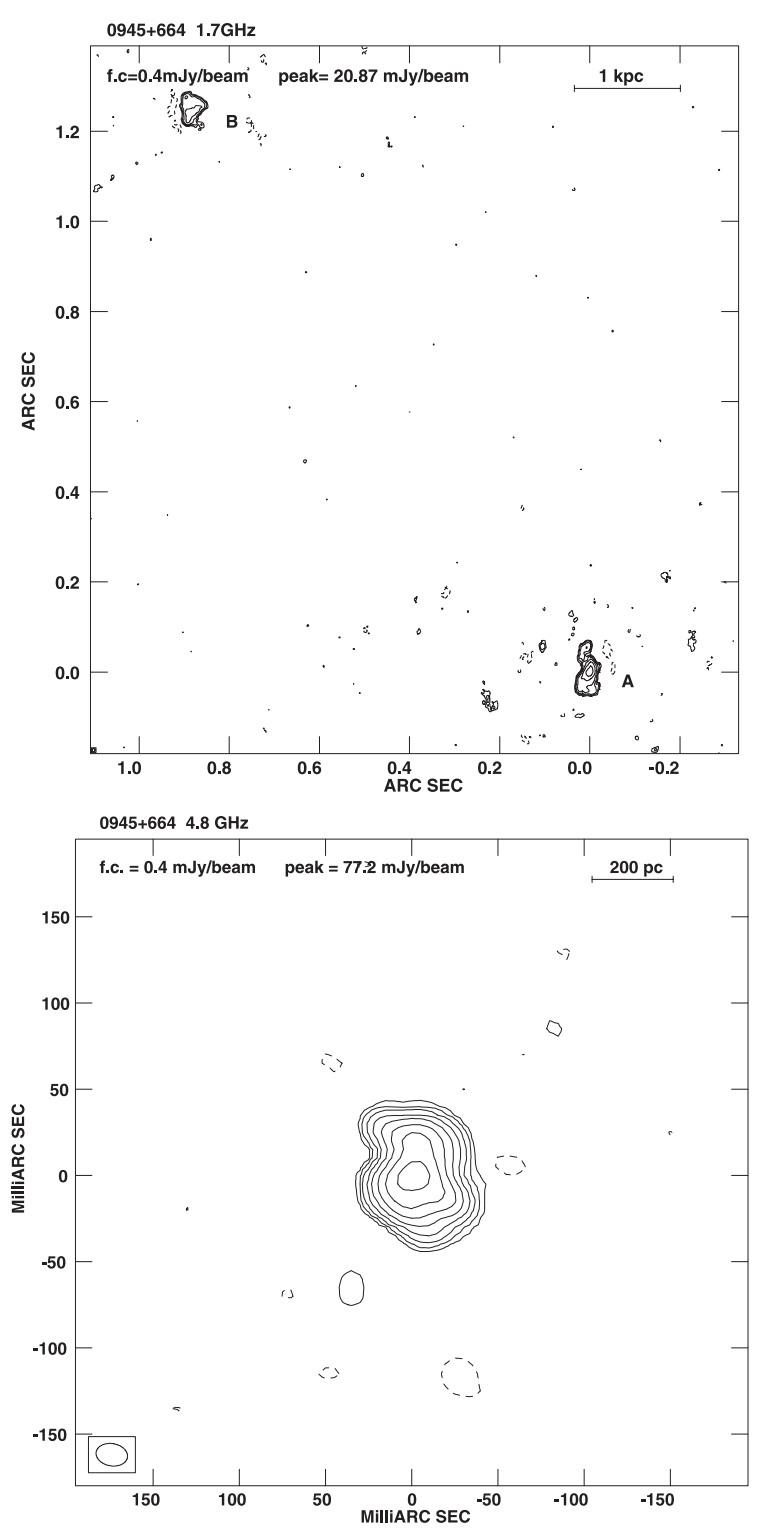

Fig. 1. continued.

Pyatunina et al. 2000) and from VLBA observations at 15, $22 \mathrm{GHz}$ (Wiik et al. 2001) and $43 \mathrm{GHz}$ (Pyatunina et al. 2000) are also available. From these images our component $A$ turned out to have a rather complex structure, being resolved into a bright component (called $A$ in Pyatunina et al. 2000) plus two weaker ones ( $B$ and $C$ in Pyatunina et al. 2000), which show activity and possibly superluminal motions.

Our images at 8.4 and $15 \mathrm{GHz}$ are consistent with the other images as far as the broad structure is concerned. The very weak south-western extension of $A$ could be related to the faint intermediate component in Kellermann et al. (1998) and component $C$ in Pyatunina et al. (2000). Compared with the $2 \mathrm{GHz}$ data of Piner et al. (1998), with a resolution similar to ours, the separation we measure at $8.4 \mathrm{GHz}$ between $A$ and $D$ strengthens its constancy over a time baseline of ten years, giving a proper motion of $0.02 \pm 0.03 \mathrm{mas} / \mathrm{yr}$, corresponding to $\beta_{\text {app }} \approx 0.3 \pm 0.44$. Furthermore, our $15 \mathrm{GHz}$ image shows a separation between $A$ and the bright features $D 1$ and $D 2$ equal to 
that reported by Kellermann et al. (2004) at the same frequency over a six-year interval, confirming that no apparent motion is present. At our epoch of observation, components $A$ and $D$ had spectral indices $\alpha_{8.4}^{15} \approx 0.32$ and $\approx 0.58$ respectively.

In our $15 \mathrm{GHz}$ image, component $A$ is slightly extended toward $D$. A two-component fit gives a bright component with a flux density $\approx 1200 \mathrm{mJy}$ that we call $A 1$ and a fainter component $\left(270 \mathrm{mJy}\right.$ at $0.65 \pm 0.20$ mas from $A 1$ in $\left.\mathrm{PA} \sim-53^{\circ}\right)$ that we call $B$. This $B$ component could be the homonymous component for which Pyatunina et al. (2000) and Kellermann et al. (2004) give proper motions of $0.18 \pm 0.01 \mathrm{mas} / \mathrm{yr}$ and $0.25 \pm 0.06 \mathrm{mas} / \mathrm{yr}$ respectively, and $\beta_{\text {app }}=2.7 \pm 0.15, \beta_{\text {app }}=3.8 \pm 0.9$ with our adopted cosmological parameters. The radial distance we measure is slightly smaller but compatible with the extrapolation from Pyatunina et al. (2000) to our observing date. Instead it is smaller at the $2.8 \sigma$ level compared with the extrapolation from Kellermann et al. (2004). This may point to a lower expansion rate. However, we note that there are differences of up to $20^{\circ}$ in the reported position angles, which may cast some doubt on the component identification or mark a rather complex situation about the source structure and/or motions. However, in Kellermann et al. (2004) component $B$ has a low rating in their quality marks.

We note that in Piner \& Kingham (1998) and in Pyatunina et al. (2000) components $A 1$ and $B$ both seem to have a flat spectrum between 8.4 and $15 \mathrm{GHz}$. Instead in the range 8.4$43 \mathrm{GHz}$ the brighter $A 1$ seems to have a transparent spectrum, while $B$ seems to be still flat. Although no errors are quoted for the flux densities and therefore the spectral indices may be uncertain, this suggests that one should not exclude the alternative interpretation that the core is component $B$ (called $C_{1}$ in Piner \& Kingham 1998) and therefore that the source could be a very asymmetric CSO. The asymmetries in component size and flux density (11:1) are not dissimilar to those seen in other very small, tens of $\mathrm{pc}$, two-sided sources.

0859+470. In our $15 \mathrm{GHz}$ image this quasar is characterized by a bright compact component and a more diffuse taillike emission extending out to $\sim 10$ mas to the North. The radio spectral index is $\alpha \sim 0.3$. As a result the object is a "classical" flat spectrum quasar.

We have compared the separations of components $A 1, A 2$, $A 3$ and $B$ with those given in Fey et al. (1997) at $8.4 \mathrm{GHz}$, at a resolution similar to our own. For that purpose we have also convolved our image to their resolution. The two observations were carried out $\approx 4.6$ years apart. Our component $B$ is very likely component 4 in Fey et al. (1997), and our component $A 3$ could be their component 2 , in which case it would have moved away from $A 1$ by $0.38 \pm 0.15$ mas with $\beta_{\text {app }}=2.8 \pm 1.1$. Their component 3 is not seen in our image and it might have moved out and now be confused with component $B$. Component $A 2$ might be a new one, not seen in 1995. Although two epochs only do not allow us to firmly determine proper motions, there is no doubt that structure changes have occurred between the two epochs.

0945+664. The spectral index of the source (Kühr et al. 1981 ) is $\approx 0.5$ at frequencies below $5 \mathrm{GHz}$ and then it steepens
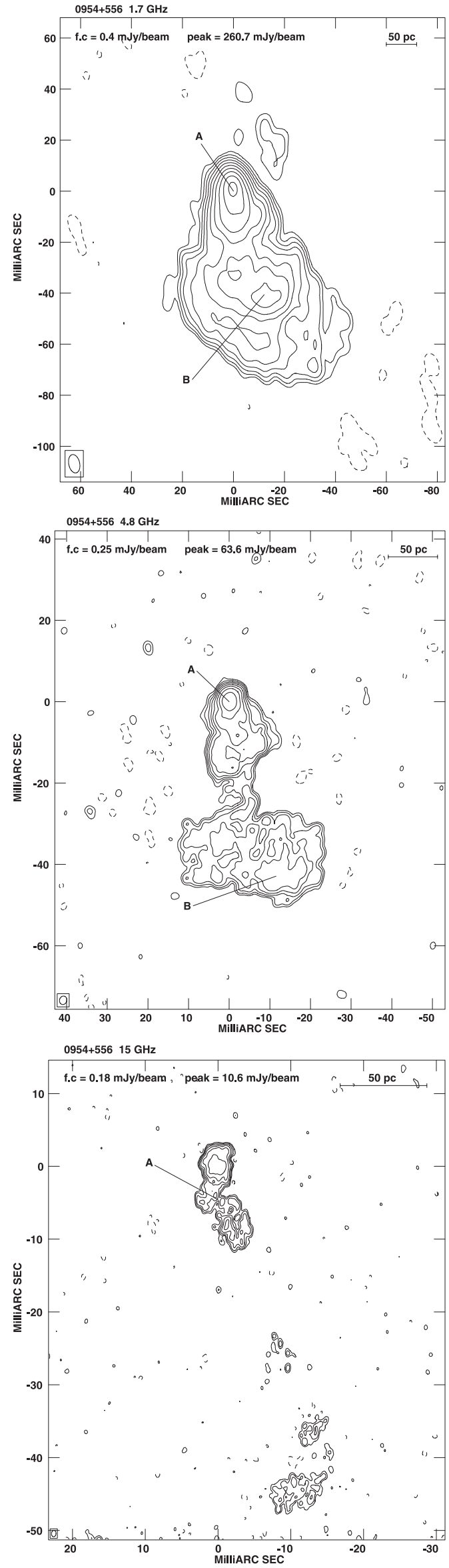

Fig. 1. continued. 
gently to $\approx 0.75$ above $10 \mathrm{GHz}$. Xu et al. (1995) presented a VLA image at $1.4 \mathrm{GHz}$, with a resolution of 1.15 , where the source is slightly resolved, possibly double, with a separation $\approx 1$.'5.

Unpublished images at $5 \mathrm{GHz}$ (MERLIN, see NED), 8.4 and $15 \mathrm{GHz}$ (VLA, J. Browne, private communication) show the double structure better and allow us to determine the spectral indices of the radio lobes (component $A$ has $\alpha_{8.4}^{15}=0.8$ and component $B \alpha_{1.7}^{4.8}=1$ ).

The image we obtained at $1.7 \mathrm{GHz}$ shows two components, $A$ and $B$, separated by $\sim 1^{\prime \prime} .5$ in PA $\sim 35^{\circ}$, well resolved by VLBI observations which are the hot spots of the two lobes. This image accounts for $20 \%$ only of the total flux density of this source.

At $4.8 \mathrm{GHz}$ we detected one component only, very likely the one stronger at $1.7 \mathrm{GHz}$ (component $A$ ), and on a small subset of short baseline only. We present in Fig. 1 a tapered image, again accounting for $20 \%$ of the source total flux density at this frequency.

At $15 \mathrm{GHz}$ also component $A$ is completely resolved out and the image revealed no significant radio emission. We conclude that the object is an MSO, even if the core component has not been identified.

0954+556. The integrated spectrum (Kühr et al. 1981) is straight up to $30 \mathrm{GHz}$, with a spectral index $\alpha \approx 0.4$ and no sign of variability. Our 1.7 and $4.8 \mathrm{GHz}$ images show radio emission distributed over two fat components separated by $\sim 50$ mas. The North component $(A)$ contains a bright compact hot spot, while the South component $(B)$ is characterized by a more diffuse emission which is resolved out at $15 \mathrm{GHz}$.

The integrated flux densities from our images account only for $60 \%, 43 \%$ and $20 \%$ of the total flux density of the source at $1.7,4.8$ and $15 \mathrm{GHz}$ respectively. Convolving the two higher frequency images to the same resolution of the one at $1.7 \mathrm{GHz}$ we have obtained the three-frequency spectrum of the northern compact hot spot. We find $\alpha \approx 0.4$.

The South component $(B)$ is characterized by a more diffuse emission, with $\alpha_{1.7}^{4.8}=0.49$. The overall structure of this object resembles that of 4C31.04 (Giroletti et al. 2003), one of the weakest known CSOs. We would classify $0954+556$ as a $\mathrm{CSO}$, even if in our images there is no indication of a feature which could be the core candidate at a level $\gtrsim 2 \mathrm{mJy} /$ beam. Its total extent is $\sim 80$ mas, corresponding to $\sim 340 \mathrm{pc}$. However, VLA images at several frequencies (Hong et al. in preparation) show on arcsecond scale an unresolved component containing the structure that we see on the tenth of arcsec scale, and two further components extending out for 2 ". 5 to North-East and $4{ }^{\prime \prime} 0$ to North-West respectively. The overall size is $\approx 4$ ". $^{\prime}$, corresponding to a linear size of $\approx 19 \mathrm{kpc}$, qualifying the arcsecond radio structure as an MSO. The missing flux density in our images is accounted for by the extended emission on arcsecond scales. In Fig. 2 we show a VLA image at $1.4 \mathrm{GHz}$, with a resolution of $\sim 1$ ". 4 , that we have produced from VLA archive data, showing the overall structure. Note how much this source recalls 3C 286 (Cotton et al. 1997).

The relation of the mas to the arcsec structure is at the moment far from being understood. It could be a case of a

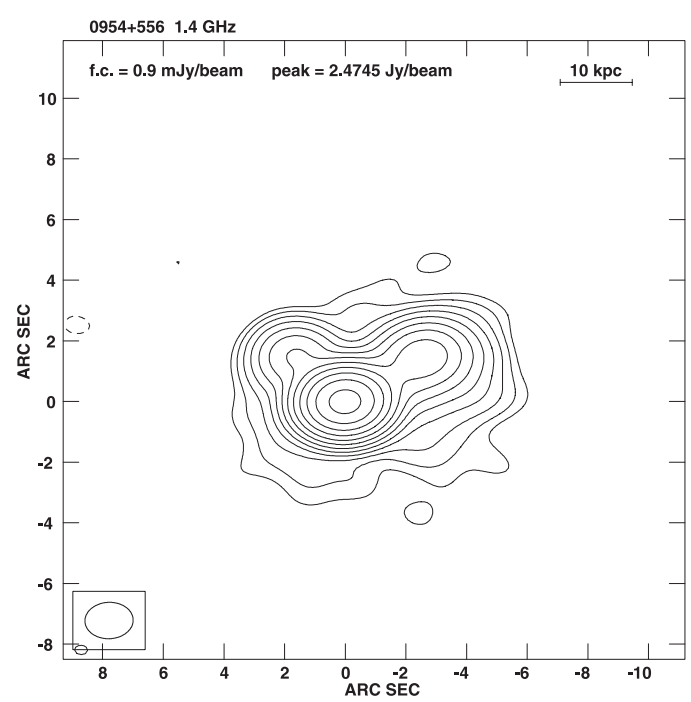

Fig. 2. $1.4 \mathrm{GHz}$ VLA images of $0954+556$ : the first contour (f.c.) is generally three times the rms noise level on the image; contour levels increase by a factor of 2; the restoring beam is shown in the bottom left corner of each image.

smothered source (Baum et al. 1990) or of recurrent activity, although neither of these possibilities appears convincing. Further investigation of this source is necessary.

\section{The list of CSOs and MSOs from the PW catalogue}

In Table 4 we give the list with relevant data compiled from the literature of all the "compact" sources from the PW catalogue which, on the basis of literature data, are not genuine core-jet radio sources. We are confident that all the CSOs/MSOs in the catalogue have been spotted and that the list is complete. Most of these sources can be classified as CSOs/MSOs. A few of them have an "anomalous" complex structure. For completeness, we also list them in the bottom section of the Table 4 . In the PW sources $0831+55$, 3C 216, 1345+125, 3C 299, $1502+10$, originally classified by the authors as $U$ (unresolved, $<2 \operatorname{arcsec}$ ), from subsequent more sensitive observations revealed additional low brightness emission. These objects are therefore extended radio sources.

\section{Conclusions}

We have presented the results of multi-frequency VLBA observations for 6 sources selected from the PW catalogue on the basis of their ambiguous morphological and spectral classification.

We confirm the core-jet structure of three of them $(0133+476,0153+744,0859+470)$, all identified as quasars. Hints for superluminal structure changes are found from a comparison with literature data. Also components with no detectable motion over long time scales are present.

One source $(0202+149)$ is, in many respects, an "enigmatic" object. Its radio structure may be that of a core-jet or a very asymmetric CSO, although we feel that further investigations are needed to firmly establish which is the radio core. 
Table 4. General parameters of PW CSOs/MSOs: Col. 1: source name; Col. 2: optical identification (Id), G = galaxy, Q = quasar, EF = empty field; Col. 3: redshift (z); Col. 4: flux density (Jy) at $2.7 \mathrm{GHz}$, from PW; Col. 5: spectral index $\alpha_{5.0}^{2.7}$, from PW; Col. 6: maximum angular size (LAS); Col. 7: frequency of spectral flux density peak; Col. 8: linear Size (LLS); Col. 9: log Radio power at 2.7 GHz; Col. 10: concise structure description: $\mathrm{sD}, \mathrm{aD}, \mathrm{dD}, \mathrm{xD}=$ symmetric, asymmetric, distorted, complex Double; c, $\mathrm{J}=\mathbf{c o r e}$, Jet(s) present; $\mathrm{T}=$ Triple, i.e. three components without an obvious source center; $\mathrm{Cx}=$ Complex. The discrimination between $\mathrm{SD}$ and $\mathrm{aD}$ is qualitative and is mostly based on the source arm length ratio (if a core is detected) or on the lobe flux density ratio Col. 11: representative references for radio structure.

\begin{tabular}{|c|c|c|c|c|c|c|c|c|c|c|c|}
\hline Source & (2) & (3) & $\begin{array}{l}S_{2.7} \\
\mathrm{Jy} \\
(4)\end{array}$ & (5) & $\begin{array}{l}\text { LAS } \\
\operatorname{arcsec} \\
(6)\end{array}$ & $\begin{array}{l}v_{\max } \\
\mathrm{GHz} \\
(7)\end{array}$ & $\begin{array}{l}\text { LLS } \\
\mathrm{kpc} \\
(8)\end{array}$ & $\begin{array}{l}\log P_{2.7} \\
\mathrm{~W} / \mathrm{Hz} \\
(9)\end{array}$ & $\begin{array}{l}\text { Alternative } \\
\text { name } \\
(10)\end{array}$ & $\begin{array}{l}\text { Radio } \\
\text { structure } \\
(11)\end{array}$ & References \\
\hline $0026+346$ & $\mathrm{G}$ & 0.52 & 1.5 & 0.28 & 0.04 & 1.31 & 0.15 & 26.59 & & $\mathrm{sD}, \mathrm{c} ?$ & $14,17,18$ \\
\hline $0116+319$ & G & 0.06 & 2.1 & 0.58 & 0.1 & 0.4 & 0.08 & 24.91 & 4C 31.04 & $\mathrm{sD}, \mathrm{c}$ & 27 \\
\hline $0127+233$ & Q & 1.46 & 1.7 & 0.70 & 2.6 & $\leq 0.1$ & 11.1 & 27.65 & $3 \mathrm{C} 43,4 \mathrm{C} 23.06$ & $\mathrm{dD}, \mathrm{c}, \mathrm{J}$ & 1,9 \\
\hline $0138+136$ & G & 0.62 & 1.5 & 0.77 & 1.0 & 0.12 & 3.85 & 26.84 & $3 \mathrm{C} 49,4 \mathrm{C} 13.10$ & $\mathrm{aD}, \mathrm{c}$ & 3,9 \\
\hline $0221+276$ & G & 0.31 & 1.7 & 0.99 & 2.5 & $\leq 0.1$ & 7.01 & 26.30 & $3 \mathrm{C} 67,4 \mathrm{C} 27.08$ & $\mathrm{aD}, \mathrm{c} ?$ & 3,6 \\
\hline $0223+341$ & Q & 2.91 & 1.8 & 0.53 & 1.1 & 0.15 & 4.04 & 28.18 & 4C 34.07 & $\mathrm{aD}$ & $2,23,25$ \\
\hline $0316+162$ & $\mathrm{G} ?$ & 1.0 & 4.9 & 0.89 & 0.3 & 0.8 & 1.28 & 27.83 & 4C 16.09, CTA21 & $\mathrm{aD}, \mathrm{c}$ & 2,25 \\
\hline $0404+768$ & $\mathrm{G}$ & 0.60 & 3.5 & 0.58 & 0.15 & 0.6 & 0.57 & 27.14 & 4C 76.03 & $\mathrm{aD}, \mathrm{c}, \mathrm{J}$ & $2,4,5,25$ \\
\hline $0428+205$ & $\mathrm{G}$ & 0.22 & 3.2 & 0.50 & 0.2 & 0.55 & 0.45 & 26.22 & & $\mathrm{aD}, \mathrm{c}, \mathrm{J}$ & 2,25 \\
\hline $0538+498$ & Q & 0.55 & 13.0 & 0.75 & 0.7 & 0.35 & 2.58 & 27.67 & $3 \mathrm{C} 147,4 \mathrm{C} 49.14$ & $\mathrm{aD}, \mathrm{c}, \mathrm{J}$ & $9,11,12$ \\
\hline $0710+439$ & G & 0.52 & 1.9 & 0.23 & 0.024 & 1.9 & 0.09 & 26.69 & & $\mathrm{aD}, \mathrm{c}, \mathrm{J}$ & $4,5,26$ \\
\hline $0945+664$ & G & 1.0 & 1.6 & 0.46 & 1.5 & 0.1 & 6.39 & 27.21 & 4C 66.09 & $\mathrm{sD} ?$ & 0,24 \\
\hline $0954+556$ & Q & 0.90 & 2.6 & 0.22 & 4.7 & $\leq 0.1$ & 19.7 & 27.26 & 4C 55.17 & $\mathrm{xD}$ & 0 \\
\hline $1031+567$ & G & 0.45 & 1.58 & 0.30 & 0.031 & 1.3 & 0.11 & 26.50 & & $\mathrm{sD?}$ & 5,23 \\
\hline $1153+317$ & Q & 0.42 & 1.7 & 0.92 & 0.9 & 0.1 & 2.96 & 26.57 & 4C 31.38 & $\mathrm{sD}$ & 20 \\
\hline $1203+643$ & G & 0.37 & 2.0 & 0.97 & 1.3 & 0.08 & 4.02 & 26.52 & $3 \mathrm{C} 268.3,4 \mathrm{C} 64.14$ & $\mathrm{aD}, \mathrm{c}$ & 3,9 \\
\hline $1225+368$ & Q & 1.97 & 1.6 & 1.17 & 0.055 & 1.0 & 0.23 & 28.11 & & $\mathrm{aD}, \mathrm{c}, \mathrm{J}$ & 2,25 \\
\hline $1250+568$ & Q & 0.32 & 1.5 & 0.61 & 1.6 & $<0.1$ & 4.57 & 26.23 & 3C $277.1,4 \mathrm{C} 56.20$ & $\mathrm{aD}, \mathrm{c}, \mathrm{J}$ & 6,9 \\
\hline $1323+321$ & $\mathrm{G}$ & 0.37 & 3.3 & 0.58 & 0.06 & 0.35 & 0.19 & 26.69 & $4 C 32.44$ & $\mathrm{sD}$ & $2,14,15$ \\
\hline $1328+307$ & Q & 0.85 & 10.3 & 0.51 & 3.8 & 0.1 & 15.8 & 27.90 & $3 \mathrm{C} 286,4 \mathrm{C} 30.26$ & $\mathrm{xD}, \mathrm{c}$ & 7,21 \\
\hline $1358+624$ & G & 0.43 & 2.7 & 0.66 & 0.05 & 0.5 & 0.17 & 26.75 & $4 C 62.22$ & $\mathrm{aD}, \mathrm{c}, \mathrm{J}$ & $2,4,25$ \\
\hline $1404+286$ & $\mathrm{G}$ & 0.08 & 1.9 & -0.78 & 0.01 & 6.0 & 0.01 & 25.07 & OQ 208 & $\mathrm{aD}, \mathrm{c}$ & $13,14,15$ \\
\hline $1413+349$ & $\mathrm{EF}$ & 1.0 & 1.7 & 0.60 & 0.06 & 0.8 & 0.26 & 27.28 & & $\mathrm{aD}, \mathrm{c}, \mathrm{J}$ & 2,25 \\
\hline $1458+718$ & Q & 0.91 & 5.3 & 0.56 & 2.2 & 0.07 & 9.26 & 27.68 & 3C 309.1, 4C 71.15 & aD.c.J & $7,10,17$ \\
\hline $1607+268$ & G & 0.47 & 2.9 & 0.89 & 0.055 & 1.1 & 0.19 & 26.90 & CTD 93 & $\mathrm{sD}$ & $17,22,19$ \\
\hline $1637+626$ & G & 0.75 & 2.2 & 1.00 & 0.38 & 0.3 & 1.54 & 27.26 & 3C $343.1,4 \mathrm{C} 62.27$ & $\mathrm{sD}$ & 3,9 \\
\hline $1819+396$ & G & 0.80 & 1.8 & 0.98 & 1.0 & 0.2 & 4.11 & 27.20 & 4C 39.56 & $\mathrm{dD}, \mathrm{c} ?$ & $2,20,24$ \\
\hline $1829+290$ & G & 0.84 & 1.9 & 0.80 & 3.0 & $<0.1$ & 12.5 & 27.23 & 4C 29.56 & $\mathrm{dD}$ & 2,20 \\
\hline $2021+614$ & G & 0.23 & 2.2 & -0.10 & 0.011 & 2.82 & 0.03 & 26.04 & & $\mathrm{sD}, \mathrm{c}$ & $14,15,16$ \\
\hline $2252+129$ & Q & 0.54 & 1.5 & 0.75 & 4.0 & $<0.05$ & 14.7 & 26.71 & $3 \mathrm{C} 455,4 \mathrm{C} 12.79$ & $\mathrm{sD}, \mathrm{c} ?, \mathrm{~J} ?$ & $7,8,20$ \\
\hline $2342+821$ & G & 0.74 & 2.3 & 0.92 & 0.18 & 0.5 & 0.73 & 27.22 & & $\mathrm{~T}$ & 2,25 \\
\hline $2352+495$ & G & 0.24 & 2.18 & 0.34 & 0.05 & 0.7 & 0.12 & 26.11 & & $\mathrm{dD}, \mathrm{c}, \mathrm{J} ?$ & $4,5,26$ \\
\hline $0134+321$ & $\mathrm{Q}$ & 0.37 & 8.97 & 0.83 & 1.5 & 0.08 & 4.64 & 27.16 & $3 \mathrm{C} 48$ & $\mathrm{Cx}$ & 30,31 \\
\hline $1328+254$ & Q & 1.06 & 4.6 & 0.63 & 0.1 & 0.05 & 0.43 & 27.77 & $3 \mathrm{C} 287$ & $\mathrm{Cx}$ & 29 \\
\hline $1600+335$ & $\mathrm{G} ?$ & 1.1 & 2.2 & 0.63 & $<0.1$ & 0.95 & $<0.43$ & 27.48 & & $\mathrm{Cx}$ & 20,25 \\
\hline $1634+628$ & Q & 0.99 & 2.7 & 0.95 & 0.25 & 0.25 & 1.06 & 27.57 & $3 \mathrm{C} 343$ & $\mathrm{Cx}$ & 3,28 \\
\hline
\end{tabular}

(0) This paper; (1) Fanti et al. (2002); (2) Dallacasa et al. (1995); (3) Fanti et al. (1985); (4) Readhead et al. (1996a); (5) Taylor et al. (1996); (6) Sanghera et al. (1995); (7) Akujor \& Garrington (1995); (8) Bogers et al. (1994); (9) Lüdke et al. (1998); (10) Wilkinson et al. (1986); (11) Junor et al. (1999); (12) Nan et al. (2000); (13) Stanghellini et al. (1997); (14) Kellermann et al. (1998); (15) Fey et al. (1996); (16) Tschager et al. (1999); (17) Fey et al. (1997); (18) Taylor et al. (1994); (19) Stanghellini et al. (2002); (20) Spencer et al. (1989) (21) Cotton et al. (1997); (22) Shaffer et al. (1999); (23) Fomalont et al. (2000); (24) Xu et al. (1995); (25) Dallacasa et al. (2004); (26) Taylor et al. (2000); (27) Giroletti et al. (2003); (28) Ren Dong et al. (1988); (29) Fanti et al. (1989); (30) Fanti et al. (1990); (31) Wilkinson et al. (1991) (32) Lister et al. (2003).

The last two objects are kpc scale MSOs. 0945+664 (whose suggested optical identification is with a faint galaxy without red-shift) has a double structure of which we detect the bright hot spots. The core is not detected. 0954+556 (a quasar) has a double structure on a tens of mas scale ( $340 \mathrm{pc})$ plus arcsec $(19 \mathrm{kpc})$ structure at a position angle roughly perpendicular to that of the small scale. The core is not detected and the connection of the small scale to the large scale structure is not understood.

We are confident that the last two sources complete the sample of CSOs/MSOs present in the PW catalogue and the complete list is given in Table 4. This sample is very useful to 
study the radio source distribution in the "Radio Power - Linear Size" plane in the range of linear sizes from $\approx 10 \mathrm{pc}$ to $\approx 20 \mathrm{kpc}$, which is an important tool to constrain source-evolution models (Baldwin 1982).

Acknowledgements. The VLBA is operated by the US National Radio Astronomy Observatory which is a facility of the National Science Foundation operated under a cooperative agreement by Associated Universities, Inc. This research has made use of the NASA/IPAC Extragalactic Database (NED) which is operated by the Jet Propulsion Laboratory, California Institute of Technology, under contract with the National Aeronautics and Space Administration. This research has also made use of the United States Naval Observatory (USNO) Radio Reference Frame Image Database (RRFID) and of data from the University of Michigan Radio Astronomy Observatory which has been supported by the University of Michigan and the National Science Foundation. This work was partly supported by the Italian Ministry for University and Research (MIUR) under grant COFIN 2002-02-8118.

\section{References}

Akujor, C. F., \& Garrington, S. T. 1995, A\&AS, 112, 235

Baldwin, J. E. 1982, in Extragalactic Radio Sources, ed. D. S. Heeschen, \& C. M. Wade (Dordrecht: Reidel), Proc. IAU Symp., 97,21

Baum, S. A., O’Dea, C. P., Murphy, D. W., \& de Bruyn, A. G. 1990, A\&A, 232, 19

Becker, R. H., White, R. L., \& Edwards, A. L. 1991, ApJS, 75, 1

Bogers, W. J., Hes, R., Barthel, P. D., et al. 1994, A\&A, 105, 91

Bondi, M., Padrielli, L., Fanti, R., et al. 1996, A\&A, 308, 415

Carvalho, J. C. 1985, MNRAS, 215, 463

Comastri, A., Fossati, G., Ghisellini, G., et al. 1997, ApJ, 480, 534

Cotton, W. D., Fanti, C., Fanti, R., et al. 1997, A\&A, 325, 479

Dallacasa, D., Fanti, C., Fanti, R., et al. 1995, A\&A, 295, 27

Dallacasa, D. 2003, PASA, 20, 79

Dallacasa, D., et al. 2004, A\&A, submitted

Fanti, C., Fanti, R., Parma, P., Schilizzi, R. T., \& van Brungel, W. J. M. 1985, A\&A, 143, 292

Fanti, C., Fanti, R., Parma, P., et al. 1989, A\&A, 217, 44

Fanti, R., Fanti, C., Schilizzi, R. T., et al. 1990, A\&A, 231, 333

Fanti, C., Fanti, R., Dallacasa, D., et al. 1995, A\&A, 302, 317

Fanti, C., Fanti, R., Dallacasa, D., et al. 2002, A\&A, 396, 801

Fey, A. L., Clegg, A. W., \& Fomalont, E. B. 1996, ApJS, 105, 299

Fey, A. L., \& Charlot, P. 1997, ApJS, 111, 95

Fomalont, E. B., Frey, S., Paragi, Z., et al. 2000, ApJS, 2000, 131, 95

Fugmann, W., \& Meisenheimer, K. 1988, A\&A, 76, 145

Giroletti, M., Giovannini, G., Taylor, G. B., et al. 2003, A\&A, 399, 889

Herbig, T., \& Readhead, A. C. S. 1992, ApJS, 81, 83

Hummel, C. A., Schalinski, C. J., Krichbaum, T. P., et al. 1988, A\&A, 204,68

Hummel, C. A., Krichbaum, T. P., Witzel, A., et al. 1997, A\&A, 324, 857

Impey, C. D., \& Tapia, S. 1990, ApJ, 354, 124

Johnston, K. J., Fey, A. L., Zacharias, N., et al. 1995, AJ, 110, 880

Junor, W., Salter, C. J., Saikia, D. J., et al. 1999, MNRAS, 308, 955

Kellermann, K. I., Vermeulen, R. C., Zensus, J. A., \& Cohen, M. H. 1998, AJ, 115, 1295

Kellermann, K. I., Lister, M. L., Homan, D. C., et al. 2004, AJ, in press

Kühr, H., Witzel, A., Pauliny-Toth, I. I. K., \& Nauber, U. 1981, A\&AS, 45, 367

Laing, R. A., Riley, J. M., \& Longair, M. S. 1983, MNRAS, 204, 151
Lister, M. L., Kellermann, K. I., Vermeulen, R. C., et al. 2003, ApJ, 584,135

Lüdke, E., Garrington, S. T., Spencer, R. E., et al. 1998, MNRAS, 299, 467

Marcaide, J. M., \& Shapiro, I. I. 1984, ApJ, 276, 56

von Montigny, C., Bertsch, D. L., Chiang, J., et al. 1995, ApJ, 440, 525

Murgia, M., \& Fanti, R. 1996, Rapporto Interno IRA, 228/96

Murgia, M., Fanti, C., Fanti, R., et al. 1999, A\&A, 345, 769

Murgia, M. 2003, PASA, 20, 19

Nan, R. D., Zhang, H. Y., Gabuzda, D. C., et al. 2000, A\&A, 357, 891

O’Dea, C. P., \& Baum, S. A. 1997, AJ, 113, 148

O’Dea, C. P. 1998, PASP, 110, 493

Owsianik, I., \& Conway, J. E. 1998, A\&A, 337, 69

Patnaik, A. R., Browne, I. W. A., Wilkinson, P. N., \& Wrobel, J. M. 1992, MNRAS, 254, 655

Peacock, J. A., \& Wall, J. V. 1981, MNRAS, 194, 331

Peacock, J. A., \& Wall, J. V. 1982, MNRAS, 198, 843

Pearson, T. J., \& Readhead, A. C. S. 1981, ApJ, 248, 61

Perlman, E. S., Padovani, P., Giommi, P., et al. 1998, AJ, 115, 1253

Phillips, R. B., \& Mutel, R. L. 1982, A\&A, 106, 21

Piner, B. G., \& Kingham, K. A. 1998, ApJ, 507, 706

Polatidis, A. G., Wilkinson, P. N., Xu, W., et al. 1995, ApJS, 98, 1

Polatidis, A. G., \& Conway, J. E. 2003, PASA, 20, 69

Pyatunina, T. B., Marchenko, S. G., Marscher, A. P., et al. 2000, A\&A, 358,451

Readhead, A. C. S., Taylor, G. B., Xu, W., et al. 1996a, ApJ, 460, 612

Readhead, A. C. S., Taylor, G. B., Xu, W., et al. 1996b, ApJ, 460, 634

Ren-Dong, N., Schilizzi, R. T., Fanti, C., et al. 1988, In The impact of VLBI on astrophysics and geophysics, ed. M. J. Reid, \& J. M. Moran, Proc. IAU Symp., 129, 119

Sanghera, H. S., Saikia, D. J., Lüdke, E., et al. 1995, A\&A, 295, 629

Shaffer, D. B., Kellermann, K. I., \& Cornwell, T. J. 1999, ApJ, 515, 558

Snellen, I. A. G., Schilizzi, R. T., Miley, G. K., et al. 2000, MNRAS, 319,445

Snellen, I. A. G., Mack, K.-H., Schilizzi, R. T., \& Tschager, W. 2003, PASA, 20, 38

Spencer, R. E., McDowell, J. C., Charlesworth, M., et al. 1989, MNRAS, 240, 657

Stanghellini, C., Bondi, M., Dallacasa, D., et al. 1997, A\&A, 318, 376

Stanghellini, C., O'Dea, C. P., Dallacasa, D., et al. 1998, A\&A, 131, 303

Stanghellini, C., Dallacasa, D., O'Dea, C. P., et al. 2002, NewAR, 46, 291

Stickel, M., Rieke, G. H., Kühr, H., et al. 1996, ApJ, 468, 556

Taylor, G. B., Vermeulen, R. C., \& Pearson, T. J. 1994, ApJS, 95, 345

Taylor, G. B., Readhead, A. C. S., \& Pearson, T. J. 1996, ApJ, 463, 95

Taylor, G. B., Marr, J. M., Pearson, T. J., \& Readhead, A. C. S. 2000, ApJ, 541, 112

Tschager, W., Schilizzi, R. T., Snellen, I. A. G., et al. 1999, NewAR, 43,681

Wiik, K., Valtaoja, E., \& Leppanen, K. 2001, A\&A, 380, 72

Wilkinson, P. N., Kus, A. J., Pearson, T. J., et al. 1986, in Quasars, ed. Govind Swarup, \& V. K. Kapahi (Dordrecht: Reidel), Proc. IAU Symp., 119, 165

Wilkinson, P. N., Tzioumis, A. K., Benson, J. M., et al. 1991, Nature, 352,313

Wilkinson, P. N., Polatidis, A. G., Readhead, A. C. S., et al. 1994, ApJ, 432, L87

Wright, A., \& Otrupcek, R. 1990, Parkes Catalogue, Australia Telescope National Facility

Xu, W., Readhead, A. C. S., Pearson, T., Polatidis, A. G., \& Wilkinson, P. N. 1995, ApJS, 99, 297 